\title{
An Improved Artificial Bee Colony Algorithm for Linear Least Squares and Constrained Optimization Problems
}

\author{
Zahraa Tariq Mohammed Taher \\ Statistics and information Department, \\ Faculty of Computer Science\& Mathematics, \\ Mosul University, Iraq.
}

\begin{abstract}
This paper proposes An Improved Artificial Bee Colony (Deb's-ABC) algorithm for solving constrained optimization problems and Nonnegative linear least squares problems. The proposed approach introduces different methods Based upon new search mechanism to balance exploration and exploitation abilities, generating initial population by using the orthogonal initialization method for achieving initial population that spread regularly over the feasible solution and to enhance the global convergence. In addition, we relax the Deb's rules by replacing the feasible solutions with the approximate feasible solutions in Deb's rules because some infeasible solutions with better objective function value and small violation may carry more important information than some feasible solutions. This algorithm is tested on several benchmark functions. Experimental results compared with a standard $\mathrm{ABC}$ and other algorithms show that the proposed algorithm is efficient and competitive algorithm for solving constrained optimization problems.
\end{abstract}

\section{General Terms}

ABC Algorithm, Deb's rules, LLS,COP .

\section{Keywords}

constrained optimization Problems ,Artificial Bee Colony, Swarm Intelligent, Least Square Error .

\section{INTRODUCTION}

Constrained optimization problem consist of engineering design ,economics, structural optimization and VLSI design[1].There are two types of optimization problems which have combined (continuous and discrete) design variables, nonlinear objective functions and nonlinear constrains [2]. The aim of this problem is to find parameter vector $\vec{x}$ that minimizes an objective function $\mathrm{f}(\vec{x})$ [3].

The objective function $f$ is represented on a search space $\mathrm{S}$ ,which is defined as a n-dimensional rectangle in $\mathrm{R}^{\mathrm{n}}(\mathrm{S} \subseteq$ $\left.\mathrm{R}^{\mathrm{n}}\right) . \mathrm{F} \subseteq \mathrm{S}$ is called feasible region and is defined by a set of $\mathrm{m}$ additional constraints $(\mathrm{m} \geq 0)$ and $\vec{x}$ is defined on feasible space $(\vec{x} \in F \in S)$.

Where :

$\mathrm{X}=\left(\mathrm{x}_{1}, \ldots, \mathrm{x}_{\mathrm{n}}\right) \in \mathrm{R}^{\mathrm{n}}$

The variable domains are limited by their lower and upper bounds:

$l_{i} \leq x_{i} \leq u_{i}, 1 \leq i \leq n$

$g_{j}(x) \leq 0$, for $j=1,2, \ldots . q$

$h_{j} \leq 0$, for $j=q+1, \ldots . m$

As well as, The algorithm of least squares (LLS) is a serious,simple and effective method for solving the approximate functions of over complex systems. Naturally, It shows when one would like to estimate values of parameters of a statistical model from measured data, which are subject to errors[4].As well as, LLS is a derivative based algorithm and the cost function can be represented by reduces the squares of errors and also frequently used in the fitting function to get the linear relationship between input and output [5], [6][7].There are two types of Least squares problems: linear least squares and non-linear least squares, which depend on whether or not the residuals are linear in all unknowns [7]. Without loss of generality, the Nonnegative Linear Least Squares (NLLS) problem can be formulated as follows:

$$
\min _{x \geq 0} f(x)=\frac{1}{2}\|A x-b\|^{2}=\frac{1}{2}(A x-b)^{T}(A x-b) \ldots
$$

Where $A \epsilon R^{m \times n}, m \geq n, \operatorname{rank}(A)=n, b \in R^{m}$

In the last time, many researchers solved constraints optimization problem and linear least squares by using traditional expensive methods .these methods suffered from slow convergence and frequently converging to local optimum solution. In a recent decade, Swarm Intelligence (SI) algorithms have shown considerable success in solving many constraints optimization problems,LLS and attracted more attention in recent years. For examples of these algorithms[8]: Genetic Algorithm (GA) [9], Particle Swarm Optimization (PSO) [10],differential evolution(DE)[11], and Artificial Bee Colony (ABC) algorithm [12] and so on.

$(\mathrm{ABC})$ algorithm depend on the foraging behaviour of honey bees for optimization problems [5].Many researchers have compared the performance of the $\mathrm{ABC}$ algorithm with the other population algorithms such as Genetic Algorithm (GA), ant colony algorithm, Particle Swarm Optimization (PSO) on unconstrained and constrained problems. Results have been shown that the $\mathrm{ABC}$ algorithm is better than other heuristic algorithms for solving unconstrained and constrained optimization problems and LLS problems.

In this paper, our approach is to applied an improved Artificial Bee Colony Algorithm (Deb's-ABC) on constraints and LLs optimization problems, by using new search mechanism to balance exploration and exploitation abilities, generating initial population by using the orthogonal initialization method [13] for achieving initial population that scattered uniformly over the feasible solution and to enhance the global convergence when producing initial population. In addition, we relax the Deb's rules by replacing the feasible solutions with the approximate feasible solutions in Deb's rules because some infeasible solutions with better objective function value and small violation may carry more useful information than some feasible solutions .This algorithm is tested on several benchmark functions where the numerical results show that the proposed (Deb's-ABC) algorithm has an outstanding performance for the optimization problems. 


\section{LITERATURE REVIEW}

Swarm Intelligence is a field of Artificial Intelligence that is used to model the collective intelligent behaviour of social swarms in nature[ 13] .It is used for solving constraints and inconstraints problems in a way that is inspired by the behaviour of real swarms or insect colonies like: ant colony, PSO Algorithm, evolutionary algorithm and Bee colony algorithm. Many researchers used swarm intelligent algorithms for solving complex problems and others improved swarm intelligent algorithms inorder to produce an efficient results for solving constraints and inconstraints problems .

In 2010, Leticia C. , Susana C. and Carlos A. present particle swarm optimization algorithm for solving general constrained optimization problems by allowing the boundary between the feasible and unfeasible regions to be explored because unfeasible solutions play an important role when trying to solve problems with active constraints so, they used shake mechanism when the percentage of unfeasible individuals is higher than $10 \%$.This algorithm provide better diversity maintenance and better exploration of constrained search spaces rather than standard PSO algorithm[14]. In 2011 ,Dervis K. and Bahriye A. modified ABC algorithm by using Deb's rules instead of selection mechanism in order to cope with the constraints and introducing a probabilistic selection that assigns probability values to feasible solutions based on their fitness values and infeasible solutions based on their violations .Results show that improved $A B C$ algorithm is efficiently for solving constraints optimization problems [3].

In 2013 , Bingqin Qiao and others propose a hybrid particle swarm optimization algorithm based on the multiplier penalty function to solve constrained optimization problems .This hybrid algorithm used the multiplier penalty function values as the fitness of particles and also made the particle swarm track the best particle and fly to the global best[15].In 2014, Xiangyu Kong and others, produced a hybrid artificial bee colony algorithm for solving nonnegative linear least squares problems.This algorithm used orthogonal initialization method to generate the initial swarmand a new search mechanism called (DE/best) is designed to balance the exploration and exploitation abilities. Numerical results demonstrate that the proposed algorithm is better than other algorithms for global optimization problems and nonnegative linear least squares problems[7]. In 2015, Yaosheng Liang and others produced an improved artificial bee colony (I$\mathrm{ABC}$ ) algorithm for COPs .This paper used a selection strategy based on rank selection and designed a search mechanism using the information of the best-so-far solution to balance the exploration and the exploitation at different stages ,as well as, introducing the approximate feasible solutions to suitably utilize the information of the infeasible solutions with better objective function value and small violation which called Deb's rules. The numerical results show that the I-ABC algorithm has an outstanding performance for the constraints optimization problems [16] .In 2015,A. J. Umbarkar and Others used a new in the area of evolutionary algorithms for solving optimization problems called Dual Population Genetic Algorithm. It solves constraints optimization problems by applying Maximum Constrains Satisfaction method by using DPGA which is a novel technique that tries to satisfy maximum constrains first and then it attempts to optimize objective function. The results are close to optimum value but fails to obtain exact optimum solution[17].In 2016, ChunFeng Wang, and Yong-Hong Zhang used a chaotic system and an opposition-based method for initial population also, they adopted a chaotic search in the best solution of the current iteration to improve the exploitation of onlooker bees. Results demonstrate that proposed algorithm is better than other standard algorithms [18].In 2017, Pintu Pal presented Hybrid and Particle Swarm Optimization (HPSO). The basic ideas behind this concept are inspired from winning match of tournament that which applied between pair wise populations in different round of the tournament using PSO .the results show that the proposed method is better than other methods in terms of different comparative parameters[19].

\section{ORIGINAL ARTIFICIAL BEE COLONY ALGORITHM (ABC)}

In 2005, Karaboga [5] invented a new heuristic population algorithm which simulate foraging behaviour waggle dance of honey bee swarm. In this algorithm, the food source refers to the solution of the optimization problem and the nectar amount of food source denotes fitness value of the associated solution. The artificial bee colony consists of three groups of bees: employed bees, onlookers and scout. The hive classified in to $50 \%$ employed bees and $50 \%$ onlooker bees. The employed bees exploring the food sources and sharing the information of the food sources with the onlooker bees. The onlooker bees will exploit one of the food sources. The probability of the food source(solution) being selected by the onlooker bee is proportional to the quality of the food[16]. The number of employed bees is equal to the number of solutions $\mathrm{SN}$ in the population.At initialization step, ABC generates initial population of SN solutions randomly using the following equation :

$x_{i j}=x_{i j}+\emptyset_{i j}\left(x_{i j}-x_{k j}\right)$

Where $k \in\{1,2, \ldots, S N\}$, and $J \in\{1,2, \ldots, D\}$. A randomly chosen index $\mathrm{k}$ has to be different from $\mathrm{I}$ and $\emptyset_{i j}$ is a random number in the range[ $-1,1]$.

These food sources are randomly assigned to SN number of employed bees and their finesses are evaluated. The search equation for employed bees and onlooker bees can be described as follows:

$x_{n e w(j)}=x_{i, j}+\emptyset_{i, j}\left(x_{i, j}-x_{k, j}\right)$

Once $x_{\text {new }}$ is obtained, a greedy selection then performs between the old and candidate solutions. In the onlooker bee step, an onlooker bee selects a food source $\mathrm{x}_{\mathrm{i}}$ depending on the probability value $\mathrm{p}_{\mathrm{i}}$ calculated as follows:

$p_{i}=\frac{f_{i}}{\sum_{i=1}^{S N} f_{i}}$

where $f_{i}$ is the fitness value of the ith food source $x_{i}$. the higher the $f_{i}$ is, the more probability that the ith food source is selected.

If a position cannot be improved through predetermined number of cycles, then the food source is assumed to be abandoned. The value of predetermined number of cycles is an important control parameter of $\mathrm{ABC}$ algorithm, which is called limit for abandonment. Assume that the abandoned source is $\mathrm{Xi}$ and $\mathrm{j} \in\{1,2, \ldots, \mathrm{D}\}$, then the scout discovers a new food source to be replaced with Xi.This operation can be defined as :

$x_{i, j}=x_{m i n, j}+\operatorname{rand}(0,1)\left(x_{\max , j}-x_{\min , j}\right)$

Original artificial bee colony algorithm

Initialize the population of solution

Evaluate the initial population cycle $=1$ 


\section{Repeat}

Employed bee phase

Apply greedy selection process

Calculate the probability values

Onlooker bee phase

Scout bee phase

Memorize the best solution achieved so far

cycle $=$ cycle +1

until cycle $=$ maximum cycle number

fig.1: show Original artificial bee colony algorithm

\section{Proposed Algorithm}

\subsection{Initial Population by using (1.1) Algorithm:}

Set of food source positions which are selected by the bees using an orthogonal array to determine a small number of combinations that are scattered uniformly over the space of all possible combinations, to provide information about the location of the solution. So that, in this paper we generate initial population by using the orthogonal initialization method [13 ][20][ 7].

Step 1.1 :

Divide the feasible solution space $[1, \mathrm{u}]$ into $\mathrm{S}$ subspaces $\left[l_{1}, \mathrm{u}_{1}\right],\left[1_{2}, \mathrm{u}_{2}\right], \mathrm{L},\left[\mathrm{l}_{\mathrm{s}}, \mathrm{u}_{\mathrm{s}}\right]$ based on the following equations:

$$
\left\{\begin{array}{c}
l_{i}=l+(i-1)\left(\frac{u(s)-l(s)}{S}\right) l_{s}, \\
u_{i}=u+(S-i)\left(\frac{u(s)-l(s)}{S}\right) l_{s}, \\
=1,2, L, S .
\end{array}\right.
$$

Here, $u(s)-l(s)=\max _{L s i s D}\left\{u_{i}-l_{i}\right\}$.

Step 1.2:

Quantize subspace $\left[1_{\mathrm{i}}, \mathrm{u}_{\mathrm{i}}\right]$ into Q1 levels based on

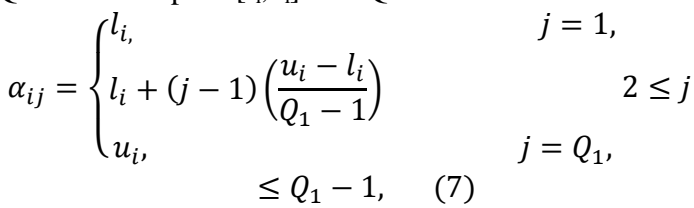

Where $Q_{1}$ is odd.Afterthat, construct orthogonal array $L_{M_{1}}\left(Q_{1}^{N}\right)=\left[a_{i j}\right]_{M_{1} * N}$ to select $\mathrm{M}_{1}$ individuals based on

$$
\left\{\begin{array}{c}
\left(\alpha_{1, a_{11}}, \alpha_{2, a_{12}}, L, \alpha_{N, a_{1 N}}\right) \\
\left(\alpha_{1, a_{21}}, \alpha_{2, a_{22}}, L, \alpha_{N, a_{2 N}}\right) \\
L \\
\left(\alpha_{1, a_{M_{1} 1}}, \alpha_{2, a_{M_{12}}}, L, \alpha_{N, a_{M 1 N}}\right)
\end{array}\right.
$$

Here, $L_{M_{1}}\left(Q_{1}^{N}\right)$ ) can be generated as follows. Select the smallest $\quad \mathrm{J}_{1} \quad$ fulfilling $\quad\left(Q_{1}^{J_{1}}-1\right) /\left(Q_{1}-1\right) \geq N \quad$.If $\left(Q_{1}^{J_{1}}-1\right) /\left(Q_{1}-1\right)=N \quad, \quad$ then $\quad \mathrm{N}^{\prime}=\mathrm{N} \quad$ else $\mathrm{N}^{\prime}=\left(Q_{1}^{J_{1}}-1\right) /\left(Q_{1}-1\right)$. Then, construct the basic columns based on $\quad j=\frac{Q_{1}^{k-1}-1}{Q_{1}-1}+1, a_{i j}=\left\lfloor\frac{i-1}{Q_{1}^{J_{1}-k}}\right\rfloor \bmod Q_{1}$, for $\mathrm{i}=1, \ldots, \mathrm{L}, \mathrm{M}_{1}, \mathrm{~K}=1, \mathrm{~L}, \mathrm{~J}_{1}$. Construct the non- basic columns as
$j=\frac{Q_{1}^{k-1}-1}{Q_{1}-1}+1, a_{j+(s-1)\left(Q_{1}-1\right)+t}=\left(a_{s} * t+a_{j}\right) \bmod Q_{1}$, for $\mathrm{s}=1, \mathrm{~L}, \mathrm{j}-1, \mathrm{t}=1, \mathrm{~L}, Q_{1}$. Thus, the orthogonal array $L_{M_{1}}\left(Q_{1}^{N^{\prime}}\right)$ is constructed. Delete the last $\mathrm{N}^{\prime}-\mathrm{N}$ columns of $L_{M_{1}}\left(Q_{1}^{N^{\prime}}\right)$ to get $L_{M_{1}}\left(Q_{1}^{N}\right)$ where $\mathrm{M}_{1}=Q_{1}^{J_{1}}$.

Step 1.3 :

Among the $M_{l} S$ individuals, select $\mathrm{SN}$ individuals having the smallest cost as the initial population.

\subsection{Search Mechanism by using Differential evolution (DE)}

The bee algorithm achieved high results in the solution of constrained optimization problems[3], and showed better performance than other algorithms like :PSO ,GA and Ant Colony Algorithms. The swarm intelligent Algorithms try to achieve balancing between exploration and exploitation abilities to check good optimization performance . exploration means the ability to search the indefinite regions in the solution space to find the global optimum, while the exploitation refers to the ability to reach better solutions based on the information of the previous good solutions. So that , the search equation in $\mathrm{ABC}$ Algorithm is not good at exploitation. In order to improve it, a new search mechanism is proposed by Differential evolution (DE) .

Differential evolution (DE) is a simple efficient evolutionary based algorithm, whose follows the general stages of an evolutionary algorithm. It depends on generating a new position for an individual by calculating vector differences between other randomly selected individuals in the population. In DE algorithm, there are three evolutionary important operations : mutation, crossover and selection. There are several types of mutation operation, which adopted differently. Among them, "DE/best/2" can improve the exploitation abilities of algorithm, which can be described as follows:

$$
\begin{aligned}
& \text { DE/best/1": Vi } \\
& =X_{\text {best }}+F(X r 1 \\
& -X r 2) \text {, } \\
& \text { 'DE/best/2": } V i=X_{\text {best }}+F(X r 1-X r 2)+ \\
& F(X r 3-X r 4), \quad(10)
\end{aligned}
$$

Where $\mathrm{i}=\{1,2, \ldots, \mathrm{SN}\}$ and $\mathrm{r}_{1}, \mathrm{r}_{2}, \mathrm{r}_{3}$ and $\mathrm{r}_{4}$ are different random integer which selected from $\{1,2, \ldots, \mathrm{SN}\} . \mathrm{x}_{\text {best }}$ is the global best solution; $F \in[0.5,1]$ is a positive real number, which is known as scaling factor or amplification factor .Depending on the property of $\mathrm{ABC}$ algorithm and two different $\mathrm{DE}$ algorithms, a new search strategies is proposed as follows :

“ABC/best/1"': $v_{i, j}=x_{\text {best }, j}+\Phi_{i, j}\left(x_{r l, j}-x_{r 2, j}\right)$,
“ABC/best/2"': $v_{i, j}=x_{\text {best }, j}+\Phi_{i, j}\left(x_{r l, j}-x_{r 2, j}\right)+\Phi_{i, j}\left(x_{r 3, j}-x_{r 4, j}\right)$,

Where the indices $r_{1}, r_{2}, r_{3}$ and $r_{4}$ are different from the base index i. $\mathrm{j} \in\{1,2, \mathrm{~L}, \mathrm{D}\}$ is a randomly selected index; $\Phi_{\mathrm{i}, \mathrm{j}}$ is a random number in the range[-1,1].Eq.(11)or Eq.(12),can choose the new candidate solution only around the best solution of the old iteration[21].

\subsubsection{Evaluate the Fitness Function}

The greedy selection is replaced by Deb's rules [10] to process the constraints: 
- Any feasible solution is preferred to any infeasible solution.

- Among two feasible solutions, the one having better objective function value is preferred.

- Among two infeasible solutions, the one having smaller constraint violation is preferred.

Our aim is to exploit the important information in infeasible solution near the optimum point more than feasible solution that far away from the optimum point .So, we use the Deb's rules by replacing the feasible solutions with the approximate feasible solutions. Here, a solution $\mathrm{x}_{\mathrm{i}}$ may be an approximate feasible solution if the penalty value vio $\left(\mathrm{x}_{\mathrm{i}}\right)$ does not exceed an adaptive control parameter $d_{t}$, which should be given a big value to allow more infeasible solutions to survive to keep the diversity of the colony at the first stage and become smaller during the iteration to guarantee the gained final solution to be the feasible solution[16]. the Formula of control parameter $d_{t}$ as follows :

$$
d_{t}=\left\{\begin{array}{c}
d_{0}\left(\frac{\alpha}{d_{0}}\right)^{\frac{t}{T_{1}},} \quad \text { if } t \leq T_{1}, \\
\propto\left(1-\frac{t}{T_{2}}\right), \quad \text { if } T_{1}<t \leq T_{2}, \\
0 \quad \text { otherwise, }
\end{array}\right.
$$

Where (t ) is the number of the current cycle, $d_{0}$ is an acceptable initial penalty value which corresponds to the median value of all the penalty values, a is a pre-set small nonnegative real number, $T_{1}$ and $T_{2}$ are the cycle numbers where the control parameter $d_{t}$ will become $\alpha$ and zero, respectively.

So, the selection mechanism between two solutions using the relaxed Deb's rules can be described as follows:

- If the one is the approximate feasible solution and the other is the infeasible solution with a penalty value exceeding $\mathrm{dt}$, the approximate feasible solution is preferred.

- If they are both approximate feasible solutions, the one having better objective function value is preferred.

- If the penalty values of them exceed dt, the one having smaller constraint violation is preferred.

\subsubsection{Calculate the probability}

In order to achieve balancing between the objective function value and the constraints in the selection progress of the probability, we can use rank selection [16] which has been used for solving the unconstrained optimization problems such as tournament selection mechansim and disruptive selection mechansim [16] [22]. The detail of the rank selection strategy can be described as follows:

- Rank the SN solutions in ascending order based on their violation degrees of the constraints $\operatorname{vio}\left(\mathrm{x}_{\mathrm{i}}\right)$ and the population denoted by $\mathrm{P}_{1}$ after ranking.

- $\quad$ Sort the former s solutions of the population $\mathrm{P}_{1}$ in ascending order according to their objective function value $\mathrm{f}\left(\mathrm{x}_{\mathrm{i}}\right)$ and the population denoted by $\mathrm{P}_{2}$ after this rank. Where, $\mathrm{s}$ is determined by

$$
s=\max \left\{i \mid \operatorname{vio}\left(x_{i}\right) \leq d_{t}, x_{i} \in P_{1}\right\}
$$

Calculate the probability $\mathrm{p}_{\mathrm{i}}$ of the solution $\mathrm{x}_{\mathrm{i}}$ by the following formula [22]:

$$
\begin{aligned}
& p_{i}=\frac{1}{S N}+a_{t} \frac{S N+1-2 i}{S N(S N+1)}, \quad i=1,2, \ldots, \mathrm{SN}, \\
& a_{t}=0.2+\frac{3 t}{4 M C N}, t=1,2, \ldots . M C N
\end{aligned}
$$

where $(t)$ represents the number of the current cycle and MCN is the maximum pre-set number of cycles.

1. If there is a $x_{i}$ (optimal solution)need to be abandoned, replace it by Eq (15) :

$$
\begin{aligned}
x_{i j}=x_{i j}+\varphi_{i j}\left(x_{k j}-x_{i j}\right) & \\
& +\left(1-\varphi_{i j}\right)\left(x_{b j}-x_{i j}\right)
\end{aligned}
$$

Where $\mathrm{k}$ is a random integer selected from $\{1, \ldots, \mathrm{i}-$ $1, \mathrm{i}+1, \ldots \mathrm{SN}\}, \mathrm{x}_{\mathrm{bj}}$ is the jth parameter of the best-sofar solution $\mathrm{x}_{\mathrm{b}}$ and $\varphi_{i j}$ is a randomly generated value in $[-1,1]$.

\section{Algorithm 1.2:show proposed algorithm}

1: Initialization: preset population size $\mathrm{SN}$ and limit by Perform Algorithm 1.1 to create an initial population $\mathrm{x}_{\mathrm{i}}, \mathrm{i} \in\{1, \cdots, \mathrm{SN}\}$.

2: Cycle $=1$

3: repeat

4: Employed bee explores $x_{i}$ to generate $v_{i}$ by Eq.(11) or by Eq.(12) .

5: Make a selection between $x_{i}$ and $v_{i}$ by the relaxed Deb's rules eq.(13).

6: Calculate the probability $\mathrm{p}_{\mathrm{i}}$ of $\mathrm{x}_{\mathrm{i}}$ based on rank selection by eq.(14).

7: Onlooker bee selects a solution $\mathrm{x}_{\mathrm{i}}$ with a probability $\mathrm{p}_{\mathrm{i}}$.

8: Onlooker bee exploits $x_{i}$ by Eq.(11) or by Eq.(12) to generate $\mathrm{v}_{\mathrm{i}}$.

9: Make a selection between xi and vi by the relaxed Deb's rules eq.(13).

10: If there is a xi need to be abandoned, replace it by Eq.(15).

11: Memorize the best-so-far solution.

12: Cycle=Cycle+1.

13: until Cycle=Maximum cycle number(MCN)

14: return the best solution.

Fig2.show proposed algorithm ( Deb's- ABC)

\section{EXPERIMENT ANALYSIS}

This article has introduced a ABC-based approach for constrained optimization problems, called ( Deb's- ABC).This approach introduces relatively many changes to a traditional $\mathrm{ABC}$ algorithm, aiming to provide better diversity maintenance and better exploration of constrained search spaces. The simulation experiments are performed on 2.40 $\mathrm{GHz}$ Core i5 with 4GB RAM using Matlab 2010a software .we used a set of 20 benchmarks problems described in [21][7] and 3 nonnegative linear least squares test problems described in[7]. 


\subsection{Experiments and comparisons:}

Do not include headers, footers or page numbers in your submission. These will be added when the publications are assembled.
5.1.1 Benchmark functions and parameter settings This paper applied 20 benchmark functions and 3 nonnegative linear least squares test problems in order to minimize them . The benchmark functions presented in Table1 are tested of dimension $\mathrm{D}=30$ and $\mathrm{D}=60$. Comparison is performed to show the performance of proposed algorithm with that of $\mathrm{ABC}$ algorithm.

Table1. Benchmark functions f1 - f 20 used in experiments. D: Dimension

\begin{tabular}{|c|c|c|}
\hline functions & D & Search range \\
\hline$f_{1}(X)=\sum_{i=1}^{D} x_{i}^{2}$ & 30 and 60 & $(-100,100)^{\mathrm{D}}$ \\
\hline$f_{2}(X)=\sum_{i=1}^{D}\left|x_{i}\right|+\prod_{i=1}^{D}\left|x_{i}\right|$ & 30 and 60 & $(-10,10)^{\mathrm{D}}$ \\
\hline$f_{3}(X)=\max _{i}\left\{\left|x_{i}\right|, 1 \leq i \leq D\right\}$ & 30 and 60 & $(-100,100)^{\mathrm{D}}$ \\
\hline$f_{4}(X)=\sum_{i=1}^{D}\left(\left\lfloor x_{i}+0.5\right\rfloor\right)^{2}$ & 30 and 60 & $(-100,100)^{\mathrm{D}}$ \\
\hline$f_{5}(X)=\sum_{i=1}^{D} i x_{i}+\operatorname{rand}[0,1)$ & 30 and 60 & $(-1.28,1.28)^{\mathrm{D}}$ \\
\hline$f_{6}(X)=\sum_{i=1}^{D}\left[x_{i}^{2}-10 \cos \left(2 \pi x_{i}\right)+10\right]$ & 30 and 60 & $(-5.12,5.12)^{\mathrm{D}}$ \\
\hline$f_{7}(X)=\sum_{i=1}^{D}\left[y_{i}^{2}-10 \cos \left(2 \pi y_{i}\right)+10\right]$ & 30 and 60 & $(-5.12,5.12)^{\mathrm{D}}$ \\
\hline$f_{8}(X)=\frac{1}{4000} \sum_{i=1}^{D} x_{i}^{2}-\prod_{i=1}^{D} \cos \left(\frac{x_{i}}{\sqrt{i}}\right)+1$ & 30 and 60 & $(-600,600)^{\mathrm{D}}$ \\
\hline$f_{9}(X)=D * 418.982887-\sum_{i=1}^{D}\left(x_{i} \sin \left(\sqrt{\left|x_{i}\right|}\right)\right.$ & 30 and 60 & $(-500,500)^{\mathrm{D}}$ \\
\hline$f_{10}(X)=-2 \exp \left(-0.2 \sqrt{\frac{1}{D} \sum_{i=1}^{D} x_{i}^{2}}\right)-\exp \left(\frac{1}{D} \sum_{i=1}^{D} \cos 2 \pi x_{i}\right)+20+e$ & 30 and 60 & $(-32,32)^{\mathrm{D}}$ \\
\hline $\begin{array}{l}f_{11}(X)=\frac{\pi}{D}\left\{10 \sin ^{2}\left(\pi y_{1}\right)+\sum_{i=1}^{D}\left(y_{i}-1\right)^{2}\left[1+\sin ^{2}\left(\pi y_{i+1}\right]+\left(y_{D}-1\right)^{2}+\right.\right. \\
\sum_{i=1}^{D} u\left(x_{i}, 10,100,4\right)\end{array}$ & 30 and 60 & $(-50,50)^{\mathrm{D}}$ \\
\hline $\begin{array}{l}f_{12}(X)=0.1\left\{\sin ^{2}\left(3 \pi x_{1}\right)+\sum_{i=1}^{D}\left(x_{i}-1\right)^{2}\left[1+\sin ^{2}\left(3 \pi y x_{i+1}\right]\right.\right. \\
\left.+\left(y_{D}-1\right)^{2}\left[1+\sin ^{2}\left(3 \pi x_{D}\right)\right]\right\}+\sum_{i=1}^{D} u\left(x_{i}, 5,100,4\right)\end{array}$ & 30 and 60 & $(-50,50)^{\mathrm{D}}$ \\
\hline$f_{13}(X)=\sum_{i=1}^{D}\left|x_{i} \cdot \sin \left(x_{i}\right)+0.1 \cdot x_{i}\right|$ & 30 and 60 & $(-10,10)^{\mathrm{D}}$ \\
\hline $\begin{array}{r}f_{14}(X)=\sum_{i=1}^{D}\left(x_{i}-1\right)^{2}\left[1+\sin ^{2}\left(3 \pi y x_{i+1}\right]+\sin ^{2}\left(3 \pi y x_{1}\right)+\right. \\
\left|x_{D}-1\right|\left[1+\sin ^{2}\left(3 \pi y x_{D}\right)\right]\end{array}$ & 30 and 60 & $(-10,10)^{\mathrm{D}}$ \\
\hline $\begin{aligned} f_{15}(X)= & \sum_{i=1}^{D}\left(\sum_{k=0}^{k \max }\left[a^{k} \cos \left(2 \pi b^{k}\left(x_{i}+0.5\right)\right)\right]\right)- \\
& D \sum_{k=0}^{k \max }\left[a^{k} \cos \left(2 \pi b^{k}\left(x_{i}+0.5\right)\right)\right] \text { where } a=0.5, b=3, k \max =20\end{aligned}$ & 30 and 60 & $(-0.5,0.5)^{\mathrm{D}}$ \\
\hline
\end{tabular}




\begin{tabular}{|l|l|l|}
\hline functions & $\mathrm{D}$ & Search range \\
\hline$f_{16}=-20 \exp \left(-0.2 \sqrt{\frac{1}{n} \sum_{i=1}^{n} x_{i}^{2}}\right)-\exp \left(\frac{1}{n} \sum_{i=1}^{n} \cos \left(2 \pi x_{i}\right)+20+e\right.$ & 30 and 60 & $(-32,32)^{\mathrm{D}}$ \\
\hline$f_{17}=\left(x_{1}-1\right)^{2}+\sum_{i=2}^{n} i\left(2 x_{i}^{2}-x_{i-1}\right)^{2}$ & 30 and 60 & $(-10,10)^{\mathrm{D}}$ \\
\hline$f_{18}=\frac{1}{4000} \sum_{i=1}^{n} x_{i}^{2}-\prod_{i=1}^{n} \cos \left(\frac{x_{i}}{\sqrt{i}}\right)+1$ & 30 and 60 & $(-600,600)^{\mathrm{D}}$ \\
\hline $\begin{array}{c}f_{19}=\sin ^{2}\left(\pi y_{1}\right)+\sum_{i=1}^{n-1}\left[\left(y_{1}-1\right)^{2}\left(1+10 \sin ^{2}\left(\pi y_{1}+1\right)\right)\right]+\left(y_{n}-1\right)^{2}(1+ \\
; y_{i}=1+\frac{x_{i}-1}{4}, i=1, L, n .,\end{array}$ & 30 and 60 & $(-10,10)^{\mathrm{D}}$ \\
\hline$f_{20}=\sum_{i=1}^{n} x_{i}^{2}+\left(\sum_{i=1}^{n} 0.5 i x_{i}\right)^{2}+\left(\sum_{i=1}^{n} 0.5 i x_{i}\right)^{4}$ & 30 and 60 & $(-5,10)^{\mathrm{D}}$ \\
\hline
\end{tabular}

\subsubsection{Comparison of Deb's $A B C$ with $A B C$ :}

This section shows the comparison between Debs's ABC and the original $\mathrm{ABC}$ algorithm. Both algorithms are used the same parameters setting in numerical experiments.The population size is 100 , limit is $0.6 * \mathrm{SN} * \mathrm{D}$ and the maximum number of generations is 1000 or 2000. The Deb's ABC algorithm runs 30 times on each function and $D=30$ and 60 and maximum number of cycle (MSN) are set to 60 .

Table 2 shows the results in terms of (best)which means the best, (worst) which means the worst, mean and (std) which means the standard deviation of function value.

Table 2. Shows Best, worst, mean and standard deviation obtained by ABC and Deb's ABC for functions f1-f10.

\begin{tabular}{|c|c|c|c|c|c|c|c|c|}
\hline & \multicolumn{4}{|c|}{$\mathbf{A B C}[23]$} & \multicolumn{4}{|c|}{ Deb's ABC } \\
\hline & Best & Mean & Worst & Std & Best & Mean & Worst & Std \\
\hline f1 & $7.62 \mathrm{e}-11$ & $2.49 \mathrm{e}-09$ & $2.75 \mathrm{e}-08$ & $5.03 \mathrm{e}-09$ & $8.66 \mathrm{e}-12$ & $8.76 \mathrm{e}-11$ & $2.00 \mathrm{e}-09$ & $4.02 \mathrm{e}-09$ \\
\hline f 2 & $0.00 \mathrm{e}+00$ & $0.00 \mathrm{e}+00$ & $0.00 \mathrm{e}+00$ & $0.00 \mathrm{e}+00$ & $0.00 \mathrm{e}+00$ & $0.00 \mathrm{e}+00$ & $0.00 \mathrm{e}+00$ & $0.00 \mathrm{e}+00$ \\
\hline f3 & $1.43 e-19$ & $7.74 \mathrm{e}-18$ & $2.34 \mathrm{e}-17$ & $6.69 \mathrm{e}-18$ & $1.43 e-19$ & $1.43 \mathrm{e}-19$ & $1.43 e-19$ & $6.69 \mathrm{e}-18$ \\
\hline f4 & $3.98 \mathrm{e}-01$ & $3.98 \mathrm{e}-01$ & $3.98 \mathrm{e}-01$ & $0.00 \mathrm{e}+00$ & $4.88 \mathrm{e}-00$ & $4.88 \mathrm{e}-00$ & $3.98 \mathrm{e}-01$ & $0.00 \mathrm{e}+00$ \\
\hline f5 & $4.29 \mathrm{e}-02$ & $2.26 \mathrm{e}-01$ & $4.97 \mathrm{e}-01$ & $1.389 \mathrm{e}-01$ & $5.00 \mathrm{e}-01$ & $5.11 \mathrm{e}-00$ & $3.99 \mathrm{e}-02$ & $0.556 \mathrm{e}-02$ \\
\hline f6 & -1 & -0.99997 & -0.99981 & $5.48 \mathrm{e}-05$ & -1 & -1 & -0.99981 & $4.55 \mathrm{e}-00$ \\
\hline f7 & 3 & 3.000014 & 3.000257 & $4.97 \mathrm{e}-05$ & 3.5543 & 3.6767 & 2.02234 & $2.55 \mathrm{e}-04$ \\
\hline f8 & -3.86278 & -3.86278 & -3.86278 & $2.32 \mathrm{e}-15$ & $4.66 \mathrm{e}-06$ & $4.87 \mathrm{e}-11$ & -3.86278 & $1.44 \mathrm{e}-11$ \\
\hline f9 & $4.65 \mathrm{e}-08$ & $4.65 \mathrm{e}-08$ & $4.65 \mathrm{e}-08$ & $1.03 \mathrm{e}-16$ & $5.77 \mathrm{e}-11$ & $5.77 \mathrm{e}-11$ & $4.65 \mathrm{e}-08$ & $0.77 \mathrm{e}-22$ \\
\hline f10 & $6.99 \mathrm{e}-17$ & $8.26 \mathrm{e}-14$ & $2.09 \mathrm{e}-12$ & $3.82 \mathrm{e}-13$ & $7.56 \mathrm{e}-16$ & $7.87 \mathrm{e}-12$ & $2.09 \mathrm{e}-12$ & $2.89 \mathrm{e}-12$ \\
\hline
\end{tabular}

As shown in Table2, the mean function values of the Deb's $\mathrm{ABC}$ algorithm are equal or closer to the optimal values, and the standard deviations are small. Results show that Deb's $\mathrm{ABC}$ algorithm has the better performance than $\mathrm{ABC}$ algorithm on constraints optimization problems except at the mean time, the two algorithms have the same mean function values on functions $\mathrm{f} 2, \mathrm{f3}$ and $\mathrm{f} 6$ and also they have the same standard deviation on functions $\mathrm{f} 2, \mathrm{f} 3$ and $\mathrm{f} 4$.So, we concluded that Deb's ABC algorithm is better than ABC algorithm in all 10 functions .

5.1.3 Comparison of Deb's ABC with other Algorithms :

Table3.The best solutions obtained by the GA, PSO, DE and Deb's ABC algorithms for last 10 benchmark problems

\begin{tabular}{|c|c|c|c|c|}
\hline functions & GA[24] & PSO[25] & DE[23] & Deb's ABC \\
\hline $\mathbf{f ~ 1 1}$ & -14.440 & -15.000 & -15.000 & -15.000 \\
\hline $\mathbf{f 1 2}$ & -0.796231 & -0.669158 & -0.472 & -0.472 \\
\hline $\mathbf{f 1 3}$ & -0.990 & -0.993930 & -1.000 & 0.9999 \\
\hline
\end{tabular}




\begin{tabular}{|l|c|c|c|c|}
\hline $\mathbf{f 1 4}$ & -30626.053 & -30665.539 & -30665.539 & -30665.539 \\
\hline $\mathbf{f 1 5}$ & 4445.33 & 5126.484 & 5126.484 & 5126.489 \\
\hline $\mathbf{f 1 6}$ & -6952.472 & -6961.814 & -6954.434 & -5564.667 \\
\hline $\mathbf{f 1 7}$ & 31.097 & 24.370 & 24.306 & 33 \\
\hline $\mathbf{f 1 8}$ & -0.095825 & -0.095825 & -0.095825 & -0.095825 \\
\hline $\mathbf{f 1 9}$ & 685.994 & 680.630 & 680.630 & 699.888 \\
\hline $\mathbf{f 2 0}$ & 9079.770 & 7049.381 & 7049.248 & 9079.770 \\
\hline
\end{tabular}

From the above table, we can see that Deb's $A B C$ is better than GA in many functions except $\mathrm{f} 11$,f14 which GA has best solutions than proposed algorithm. Also, in problems f18 and f20 GA has the same solution of Deb's ABC. PSO algorithm has an equal solutions with the Deb's $\mathrm{ABC}$ in problems f11,f14 and f18.as well as, DE algorithm has the same solutions with the Deb's ABC in functions f11,f12,f14 and f18 so, we concluded that proposed algorithm has best solutions from others algorithms in most cases .

\subsection{Experiments on NLLS problems :}

\subsubsection{Test problems:}

In order to show the implementation and efficiency of the Deb's ABC algorithm that proposed in this paper, we applied three NLLS(non-linear least square) problems[7]. The 3 NLLS are following :
NLLS 1: Consider the following NLLS problem, where

$$
\boldsymbol{A}=\left[\begin{array}{ccc}
1 & 1 & 1 \\
0 & 4 & -1 \\
2 & -2 & 1
\end{array}\right], b=\left(\begin{array}{l}
6 \\
5 \\
1
\end{array}\right)
$$

The optimal solution is $x^{*}=(1,1,3)^{T}$.

NLLS 2: Let be a matrix whose diagonal elements are 500 and the non-diagonal elements are chosen randomly from the interval such that $A$ is symmetric. Let $b=A e$ where $e$ is $n \times 1$ vector whose elements are all equal to unity such that $x^{*}=(1,1, \ldots \ldots, 1)^{T} \in R^{n}$ is the unique solution .

NLLS 3:Let the matrix $A$ is given by $a_{i, i}=4 n, a_{i, i+1}=a_{i+1, i}=n$, $\mathrm{a}_{\mathrm{i}, \mathrm{j}}=0, \mathrm{i}=1,2, \ldots . \mathrm{n}$. let $\mathrm{b}=\mathrm{Ae}$. Thus the unique solution is $x^{*}=(1,1, \ldots \ldots, 1)^{T} \in R^{n}$.

Table.4. The statistical results for 30 runs tested on given 3 NLLS problems

\begin{tabular}{|l|c|l|l|l|l|c|c|c|}
\hline & \multicolumn{3}{|c|}{ ABC } & \multicolumn{3}{c|}{ Deb's ABC } \\
\hline functions & Best & Mean & Worst & Std & Best & Mean & Worst & Std \\
\hline NLLS1 & $2.36 \mathrm{e}-17$ & $2.65 \mathrm{e}-16$ & $7.49 \mathrm{e}-16$ & $1.87 \mathrm{e}-16$ & $8.00 \mathrm{e}-12$ & $2.55 \mathrm{e}-16$ & $2.44 \mathrm{e}-14$ & $1.66 \mathrm{e}-12$ \\
\hline NLLS2 & $7.98 \mathrm{e}+01$ & $2.53 \mathrm{e}+02$ & $7.47 \mathrm{e}+02$ & $1.69 \mathrm{e}+02$ & $7.99 \mathrm{e}+01$ & $2.78 \mathrm{e}+01$ & $5.44 \mathrm{e}+08$ & $1.69 \mathrm{e}+02$ \\
\hline NLLS3 & $4.93 \mathrm{e}+03$ & $1.69 \mathrm{e}+04$ & $3.77 \mathrm{e}+04$ & $9.27 \mathrm{e}+04$ & $3.15 \mathrm{e}+00$ & $3.00 \mathrm{e}+03$ & $3.60 \mathrm{e}+03$ & $4.32 \mathrm{e}+00$ \\
\hline
\end{tabular}

In table 4,we can see that proposed algorithm is better than classical ABC in NLLS1 and NLLS2 except NLLS3 in best term .From the other side the Deb's ABC has best standard deviation in all NLL problems except NLL2 which has the same value of our proposed algorithm .

\section{CONCLUSION}

This paper proposes an improved Artificial Bee Colony algorithm based on the Deb's rules, Differential evolution Algorithm like DE/best1 and DE/best2 to balance exploration and exploitation abilities, as well as , using the orthogonal initialization method for achieving initial population that spread regularly over the feasible solution and to enhance the global convergence to solve constrained optimization problems . To verify the performance of the proposed algorithm, a set of 20 test functions and 3 nonnegative linear least squares test problems are used in the experiments. Comparison of Deb's $\mathrm{ABC}$ with other algorithms like $\mathrm{ABC}, \mathrm{PSO}, \mathrm{GA}$ and DE algorithms. Therefore, results demonstrate that the Deb's ABC algorithm proposed in our paper is more effective for constraints optimization and NLLS problems.

\section{REFERENCES}

[1] Konstantinos E. Parsopoulos and Michael N. Vrahatis," Particle Swarm Optimization Method for Constrained Optimization problems", in:proceedings of the EuroInternational Symposium on Computational Intelligence 2002 ,Press,2002,pp.214-220.Ding, W. and Marchionini, G. 1997 A Study on Video Browsing Strategies. Technical Report. University of Maryland at College Park.

[2] Ivona BRAJEVIC, Milan TUBA, Milos SUBOTIC," Improved Artificial Bee Colony Algorithm for Constrained Problems", Recent Advances In Neural Networks, Fuzzy Systems \& Evolutionary Computing .

[3] Dervis Karaboga, Bahriya Akay, "A modified Artificial Bee Colony (ABC) algorithm for Constrained optimization problems", Applied Soft Computing ,2011.

[4] W. Gander et al., Scientific Computing - An Introduction using Maple and MATLAB, Texts in Computational Science and Engineering 11, DOI 10.1007/978-3-319- 
04325-8 6, (c) Springer International Publishing Switzerland 2014

[5] Dervis KARABOGA, "An Idea Based On Honey Bee Swarm for Numerical Optimization”, Technical Report TR06, Erciyes University, Engineering Faculty, Computer Engineering Department, 2005.Brown, L. D., Hua, H., and Gao, C. 2003. A widget framework for augmented interaction in SCAPE.

[6] Dervis Karaboga, Bahriye, Akay, "A comparative study of artificial bee colony algorithm", Applied Mathematics and Computation, 214(1), 2009, pp. 108-132.

[7] Xiangyu Kong, Sanyang Liu and Zhen Wang "An Effective Hybrid Artificial Bee Colony Algorithm for Nonnegative Linear Least Squares Problems", Journal of Engineering Science and Technology Review 7 (3) (2014) 96 - 107,2014 .

[8] Soudeh Babaeizadeh and Rohanin Ahmad," An Efficient Artificial Bee Colony Algorithm for Constrained Optimization Problems", Journal of Engineering and Applied Sciences, 2014 .

[9] Deb K (2000) An efficient constraint handling method for genetic algorithms. Comput Method Appl M 186(2):311-338.

[10] Hu X, Eberhart R (2002) Solving constrained nonlinear optimization problems with particle swarm optimization. In: Proceedings of the sixth world multiconference on systemics, cybernetics and informatics, Orlando, pp 203206 .

[11] R. Storn, K. Price ,Differential evolution-a simple and efficient heuristic for global optimization over continuous spaces, Journal of Global Optimization 23(2010)689-694.

[12] Karaboga D, Basturk B (2007) Artificial bee colony (abc) optimization algorithm for solving constrained optimization problems. In: Foundations of fuzzy logic and soft computing, Springer, pp 789-798 .

[13] Kong, X., et al. , "Hybrid Artificial Bee Colony Algorithm for Global Numerical Optimization", Journal of Computational Information Systems, 8(6), 2012, pp. 2367-2374.

[14] Leticia Cagninaa, Susana Esquivela and Carlos A.," Solving constrained optimization problems with a hybrid particle swarm optimization algorithm", Engineering Optimization Vol. 43, No. 8,August 2011, 843-866.

[15] Bingqin Qiao, Xiaoming Chang, Mingwei Cui, Kui Yao," Hybrid particle swarm algorithm for solving nonlinear constraint optimization problems", Wseas Transactions On Mathematics, Issue 1, Volume 12, January 2013.

[16] Yaosheng Liang, Zhongping Wan, Debin Fang," An improved artificial bee colony algorithm for solving constrained optimization problems", Springer 2015.

[17] A. J. Umbarkar, M. S. Joshi, P. D. Sheth," Dual Population Genetic Algorithm for Solving Constrained Optimization Problems", I.J. Intelligent Systems and Applications, 2015, 02, 34-40 .

[18] Chun-Feng Wang, and Yong-Hong Zhang," An Improved Artificial Bee Colony Algorithm for Solving Optimization Problems", IAENG International Journal of Computer Science, 27 August 2016.

[19] Pintu Pal," A Hybrid Particle Swarm Optimization Algorithm for Solving Optimization Problem", (IJCSIT) International Journal of Computer Science and Information Technologies, Vol. 8 (2) , 2017, 187-189.

[20] Liang, Jing J., Qin, A. K., Suganthan, Ponnuthurai Nagaratnam, Baskar, S., "Comprehensive learning particle swarm optimizer for global optimization of multimodal functions", IEEE Transactions on Evolutionary Computation, 10 (3), 2006, pp. 281-295

[21] Weifeng Gao*,San yang Liu,Ling ling Huang," Aglobal best artificial bee colony algorithm for global optimization", ScienceDirect Journal of Computational and Applied Mathematics.

[22] Bao L, Zeng JC (2009) Comparison and analysis of the selection mechanism in the artificial bee colony algorithm. In: Proceedings of IEEE international conference on hybrid intelligent systems, Shenyang, pp 411-416

[23] Karaboga D, Akay B (2011) A modified artificial bee colony (abc) algorithm for constrained optimization problems. Appl Soft Comput 11(3):3021-3031 .

[24] Mezura-Montes E, Coello CAC (2005) A simple multimembered evolution strategy to solve constrained optimization problems. IEEE Trans Evol Comput 9(1):117 .

[25] Mun oz Zavala AE, Aguirre AH, Villa Diharce ER (2005) Constrained optimization via particle evolutionary swarm optimization algorithm (peso). In: Proceedings of the 2005 conference on genetic and evolutionary computation, USA, pp 209-216. 